\title{
A Case of Secondary Iris Cyst AfterPupilloplasty*
}

\author{
Ja Young Lee ${ }^{1}$, Sun Woong Kim², Eun Suk Koh ${ }^{3}$, Jee Ho Chang ${ }^{1 \#}$ \\ ${ }^{1}$ Department of Ophthalmology, College of Medicine, Soonchunhyang University, Bucheon, Korea; ${ }^{2}$ Department of Ophthalmology, \\ Hando Hospital, Ansan, Korea; ${ }^{3}$ Department of Pathology, College of Medicine, Soonchunhyang University, Bucheon, Korea. \\ Email: jhchang@schbc.ac.kr
}

Received November $23^{\text {rd }}, 2012$; revised December $24^{\text {th }}, 2012$; accepted January $3^{\text {rd }}, 2012$

\begin{abstract}
Purpose: To investigate the pathogenesis of a secondary iris cyst with an immunohistochemical method. Methods: Single observational case report. A pathologic specimen was obtained from a 5-year-old girl who was found to have a secondary iris cyst. She had a history of previous penetrating ocular trauma and subsequent cataract surgery and pupilloplasty. Immunohistochemical staining with cytokeratin (CK) 19 and CK3 was used. Results: After immunohistochemical staining, the inner wall showed positive staining for CK19, which is specific for limbal, peripheral cornea and conjunctival epithelium, and negative staining for CK3, which is specific for corneal epithelium. Conclusions: With the aid of immunohistochemical analysis, a conjunctival epithelial origin was indicated, and pupilloplasty was identified as the causal event of the iris cyst while the possibility of primary iris cyst was ruled out.
\end{abstract}

Keywords: Immunohistochemistry; Pupilloplasty; Secondary Iris Cyst

\section{Introduction}

Iris cysts can be classified as primary or secondary. Primary cysts are thought to be of neuroepithelial origin, whereas secondary cysts may result from implantation, metastasis, parasites, or miotic therapy. Secondary iris cysts are usually associated with surgery or penetrating ocular injuries [1]. Although secondary cysts are generally considered to be driven by epithelial downgrowth [2], the specific origin of the cyst in our case was difficult to differentiate on the basis of light and electron microscopic characteristics.

Here, we present a secondary iris cyst whose origin was presumed to be associated with pupilloplasty by pathologic examination and an immunohistochemical study.

\section{Case Report}

A 5-year-old girl was scheduled for detailed examination under general anesthesia for a suspected solid iris mass in her right eye. She had a corneoscleral laceration with a prolapsed iris from the 8- to 10-o'clock positions 3 years ago. Two years after the primary repair, extracapsular cataract extraction with intraocular lens implantation and pupilloplasty was performed with 10 - 0 polypropylene sutures. After 8 months, her corrected visual acuity drop-

\footnotetext{
*Conflicts of Interest and Source of Funding: No conflicts of interest are declared.

${ }^{\#}$ Corresponding author.
}

ped from 20/32 to 20/63, her intraocular pressure was 20 $\mathrm{mmHg}$, and she had severe photophobia. On slit-lamp examination, which was not thorough due to poor cooperation, an iris mass nearly obstructing the visual axis was noted; the patient was then scheduled for detailed examination under general anesthesia. Under the operating microscope, an iris cyst was found stemming from the previous pupilloplasty site and extending superiorly to the 12 o'clock position and inferiorly to the 6 o'clock position, apposing at the center of the pupil (Figure 1). To secure the visual axis, we performed partial excision of the wall along the border of anterior and posterior pigment epithelium and obtained a histopathologic specimen. The well positioned intraocular lens and flat retina were noticed at the end of surgery. At postoperative 2 months, her uncorrected visual acuity was 20/32, and there was no recurrence of iris cyst up to 3 years.

With hematoxylin and eosin staining, the outer wall of the cyst was seen to consist of iris pigment epithelium containing melanin pigment granules and the inner wall was found to be stratified squamous epithelial cells (Figure 2(A)). On immunohistochemical staining, the inner wall showed positive staining for cytokeratin (CK) 19 (monoclonal, 1:100; Dako, Glostrup, Denmark), which is specific for limbal, peripheral corneal and conjunctival epithelium (Figure 2(B)), negative staining for CK3 (monoclonal, 1:100; Biotechnology, Santa Cruz, Calif.), which is specific for corneal epithelium (Figure 2(C)), and vimentin (monoclonal, predilution; Dako, Glostrup, 
Denmark), which is specific for cells of neuroectodermal origin (not shown). The outer wall of the cyst, which was thought to be posterior iris pigment epithelium, was stained with glial fibrillary acidic protein (GFAP, polyclonal, predilution; Dako, Glostrup, Denmark), which is specific for cells of neuroectodermal origin (Figure 2(D)). Consequently, these findings indicate a conjunctival epithetlial origin.

\section{Discussion}

Experimental studies have shown that the introduction of either corneal or conjunctival epithelium into the anterior chamber can lead to cyst formation [3]. Most commonly, corneal epithelium can induce the iris cysts associated with epithelial downgrowth.In this case, there were several possible origins of the cyst, including not only cor-

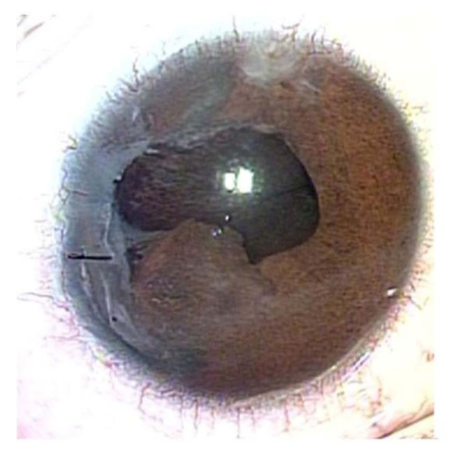

(A)

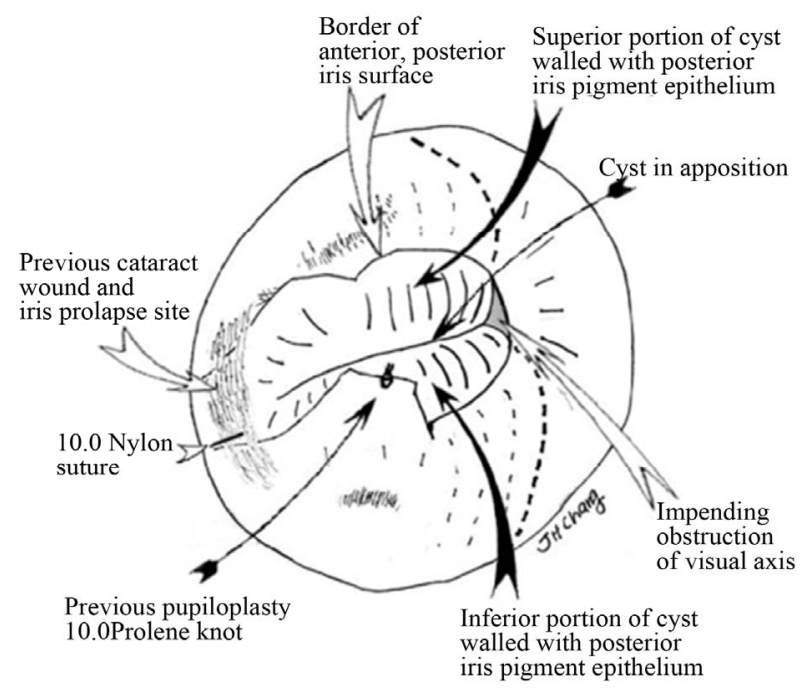

(B)

Figure 1. (A) An anterior segment image before surgery; (B) Schematic drawing of the image. An iris cyst stemming from the previous iris prolapse site and extending superiorly to 12 o'clock and inferiorly to 6 o'clock, apposing at the center of the pupil and leaving a small aperture for light to go through. The iridocorneal touch was noticed, especially at temporal cornea.

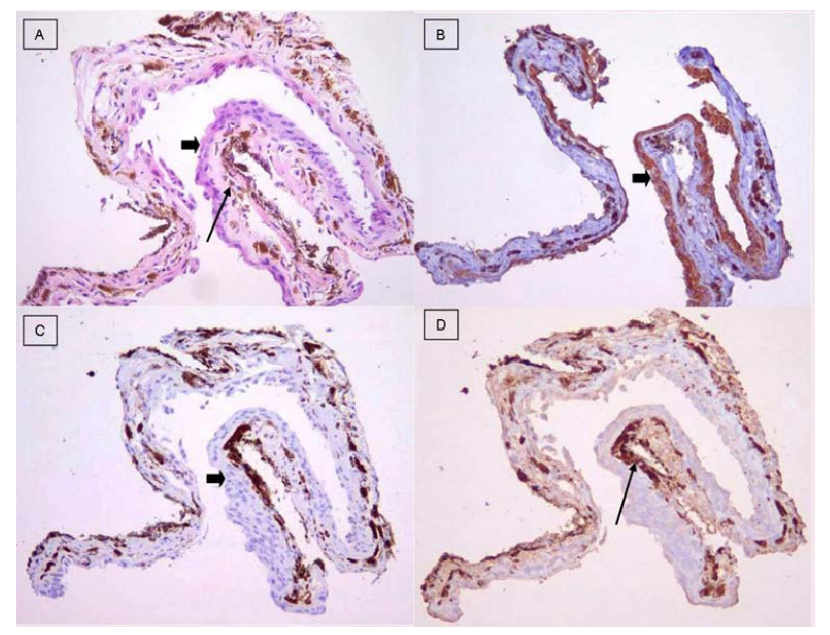

Figure 2. An obtained histopathologic specimen after surgical excision. (A) The long arrow points to the outer wall of the cyst, which consisted of iris pigment epithelium containing melanin pigment granules, and the short arrow points to the inner wall, which consisted of stratified squamous epithelial cells. Hematoxylin and eosin staining; originnal magnification, $\times 100$; (B) The arrow points to the inner wall, which showed positive staining for cytokeratin (CK) 19. (C) The arrow points to the inner wall, which showed negative staining for CK3; (D) The arrow points to the outer wall, which showed positive staining for glial fibrillary acidic protein (GFAP). (B-D) Immunohistochemical staining; original magnification, $\times \mathbf{1 0 0}$.

neal and conjunctival epithelia but also ciliary epithelium and the iris.

The intraocular implantation of epithelial cells depends on a favorable environment in the eye, which is provided by the vascular iris [4]. The epithelial cells proliferate and extend into the adjacent ocular structures, spreading anteriorly over the angle and posteriorly into the ciliary body and beyond. In the molecular pathogenesis of epithelial iris cyst evolution, membrane proteins such as ezrin, which are responsible for maintaining iris pigment epithelium integrity, may become disrupted by inflammation or intraocular manipulation, leading to cyst development [5].

Various surgical procedures including cataract surgery, penetrating keratoplasty, and radial keratotomy may predispose to the formation of an epithelial inclusion cyst. The pathogenesis of any ocular surgery is presumably related to the seeding of the epithelial cells into the iris stroma during the procedure. Several candidate procedures could have caused the iris cyst in our case. Our clinical examination revealed that the iris cyst was found stemming from the previous pupilloplasty site, not from the site of corneoscleral laceration or the corneal incision margin of the cataract surgery.We suspect that the procedure of pupilloplasty might have induced the iris cyst rather than the primary closure of the wound or cataract surgery. 
Therefore, ophthalmic surgeons should attempt to carefully manipulate the iris, even when performing a simple procedure like pupilloplasty, to prevent secondary iris cyst formation.

\section{REFERENCES}

[1] F. A. Marigo and P. T. Finger, "Anterior Segment Tumors: Current Concepts and Innovations," Survey of Ophthalmology, Vol. 48, No. 6, 2003, pp. 569-593. doi:10.1016/j.survophthal.2003.08.001

[2] M. Küchle and W. R. Green, "Epithelial Ingrowth: A Study of 207 Histopathologically Proven Cases," German Journal of Ophthalmology, Vol. 5, No. 4, 1996, pp. 211-
223.

[3] D. C. Cogan, "Experimental Implants of Conjunctiva into the Anterior Chamber," American Journal of Ophthalmology, Vol. 39, No. 4, 1955, pp. 165-172.

[4] J. V. Jester, R. A. Villasenor and J. Miyashiro, "Epithelial Inclusion Cysts Following Radial Keratotomy,” Archives of Ophthalmology, Vol. 101, No. 4, 1983, pp. 611-615. doi:10.1001/archopht.1983.01040010611018

[5] T. Kivela, J. Jaaskelainen, A. Vaheri and O. Carpen, "Ezrin, a Membrane-Organizing Protein, as a Polarization Marker of the Retinal Pigment Epithelium in Vertebrates," Cell and Tissue Research, Vol. 301, No. 2, 2000, pp. 217-223. doi:10.1007/s004410000225 